\title{
New Data Completing the Spectrum of the Ma,RMia, and RMja Genes for Resistance to Root-Knot Nematodes (Meloidogyne spp.) in Prunus
}

\author{
Henri Duval, ${ }^{1}$ Cyril Van Ghelder, ${ }^{2}$ Ulysse Portier, ${ }^{2}$ Carole Confolent, ${ }^{1}$ Pablo Meza, ${ }^{3}$ and Daniel Esmenjaud ${ }^{2, \dagger}$ \\ ${ }^{1}$ Unité de Génétique et Amélioration des Fruits et Légumes (GAFL), INRA, Montfavet, France; ${ }^{2}$ INRA, Université Nice Côte d'Azur, CNRS, \\ ISA, France; and ${ }^{3}$ Instituto de Investigaciones Agropecuarias, INIA, Centro Regional La Platina, Santiago, Chile \\ Accepted for publication 24 September 2018.
}

\begin{abstract}
Root-knot nematodes (RKN) (Meloidogyne spp.) are worldwide pests that affect a considerable number of plants, among which stone fruit (Prunus spp.) are severely attacked. Prevalent RKN species are Meloidogyne arenaria, M. incognita, and M. javanica in stone fruit but the emergent $M$. ethiopica and $M$. enterolobii are also reported to challenge perennial crops. In Prunus spp., the complete-spectrum resistance $(R)$ gene $M a$ from plum and the more restricted-spectrum $R$ genes RMia from peach and RMja from almond completely inhibit nematode multiplication and gall formation of the RKN species that they control. This study aimed to update the resistance spectra of these three major genes by evaluating their activity toward one isolate of the yet-untested RKN species mentioned above. To state whether a given gene controls a particular species, the principle of our experiment was to genotype with appropriate markers a number of individuals

segregating for this gene and then to phenotype these individuals. A perfect matching of the genotype and the phenotype of individuals indicates that the gene of interest is active against and, thus, controls the corresponding isolate of this RKN species. Segregating materials used were an $M a$ F1 plum progeny, an RMia F2 peach progeny, and an RMja F2 almond progeny. In addition to previous data, our results establish a clear spectrum for each of the three genes toward isolates from both the three prevalent species and the two emerging species. Ultimately, our results reveal that (i) $M a$ controls all of them, (ii) $R M j a$ controls all species except $M$. incognita and M. floridensis, and (iii) RMia controls $M$. arenaria, $M$. incognita, and M. ethiopica but not $M$. javanica or M. enterolobii. Our data should have wide implications for RKN resistance management and breeding and for deciphering the molecular mechanisms of the spectrum of RKN $R$ genes.
\end{abstract}

Root-knot nematodes (RKN) (Meloidogyne spp.) are major crop pests throughout the world (Jones et al. 2013; Lamberti 1979). Meloidogyne arenaria, M. incognita, and M. javanica, the predominant RKN species under Mediterranean and hot climates, are highly polyphagous and reproduce through mitotic parthenogenesis (Triantaphyllou 1985) on hundreds of cultivated and wild plant species (De Guiran and Netscher 1970). Several RKNs have been described and reported recently as emerging species with a putatively high impact on woody crops (Saucet et al. 2016). These species include the invasive $M$. ethiopica, prevalent in Chile (Carneiro et al. 2007a; Meza et al. 2016) and Brazil (Correa et al. 2014), where new intensive perennial crops such as stone fruit and kiwi are set up. These RKNs also include the polyphagous species of tropical origin, M. enterolobii (formerly M. mayaguensis), which is uncontrolled by the $\mathrm{Mi}-1$ gene from tomato and highly aggressive toward many plants (Castagnone-Sereno 2012; Rammah and Hirschmann 1988; Saucet et al. 2016; Yang and Eisenback 1983), including perennial guava crops (Carneiro et al. 2006; Carneiro et al. 2007b). Another species is M. floridensis, detected first in Prunus spp. in the southeastern United States and designated as the

†Corresponding author: D. Esmenjaud; E-mail: daniel.esmenjaud@inra.fr

Funding: Main financial support was provided by the French Ministry of Agriculture and Food (CASDAR project 'Pyrédune' number C2011-01 [20112014]) and the contract 'Prunus rootstocks' (2015-2020) between INRA and CEP Innovation (Lyon, France). Complemental funding was from the INRA BAP department (projects IVD2 and IVD3) and the Plant-Nematode Interaction team at INRA-ISA in Sophia Antipolis, France.

*The $\boldsymbol{e}$-Xtra logo stands for "electronic extra" and indicates that one supplementary table is published online.

The author(s) declare no conflict of interest.

This article is in the public domain and not copyrightable. It may be freely reprinted with customary crediting of the source. The American Phytopathological Society, 2019. peach RKN because it attacks peach resistance sources (Handoo et al. 2004; Saucet et al. 2016).

The genus Prunus is divided into five main subgenera and comprises more than 400 species distributed worldwide (Rehder 1947). Though the plum species range from diploid to hexaploid $(2 \mathrm{n}=6 \mathrm{x}=48)$, most species are diploid $(2 \mathrm{n}=2 \mathrm{x}=16)$ (Salesses et al. 1992). A majority of fruit-producing and rootstock species belong to the subgenera Amygdalus (almond and peach) and Prunophora (plums and apricot). In stone fruit crops, Prunus spp. rootstock material is predominantly susceptible to RKNs. Resistance sources have been sought from a wide range of Prunus crops with the objective of controlling these pests (Saucet et al. 2016) and they express different resistance spectra (Esmenjaud et al. 1997, 2009; Scotto La Massese et al. 1984).

Genes conferring RKN resistance have been identified especially in plum $(M a)$, peach (RMia), and almond (RMja). The major dominant gene $M a$ from Myrobalan plum (Prunus cerasifera) confers a high-level resistance to all tested RKN species (M. incognita, M. arenaria, M. javanica, M. floridensis, and M. enterolobii) (Lecouls et al. 1997; Rubio-Cabetas et al. 1999). $M a$, located on the chromosome 7 of the peach (P. persica) genome, the reference sequence for Prunus spp. (Verde et al. 2013), belongs to the toll interleukin 1 receptor nucleotide-binding leucine-rich repeat (TNL) class of genes (Claverie et al. 2011). The RMia gene carried by the peach cultivars Nemaguard and Nemared (Ramming and Tanner 1983) confers resistance to M. incognita and $M$. arenaria. Additionally, several genetic hypotheses have been put forward regarding the control of $M$. javanica in these accessions: two independent genes (Sharpe et al. 1969) or a single gene (Lu et al. 1998; Yoshida and Seike 1981). Nevertheless, the genetic determinants of this resistance remain to be identified and studies have proposed that resistance $(R)$ genes for resistance to $M$. incognita might have a partial effect on $M$. javanica (Lu et al. 1998, 1999). RMia is a putative TNL gene which is located on chromosome 2 (Claverie et al. 2004a; Duval et al. 2014). Finally, the 
RMja gene (Van Ghelder et al. 2010) in the Alnem series (Alnem1, Alnem88, and Alnem201) of bitter almond (P. dulcis) confers a specific resistance to M. javanica (Kochba and Spiegel-Roy 1975) (Table 1). RMja belongs to the same cluster as the $M a$ gene and is presumably its ortholog (Van Ghelder et al. 2018).

This article considers the five RKN species $M$. incognita, $M$. javanica, $M$. arenaria, $M$. enterolobii, and $M$. ethiopica. It aims to supplement the data on the spectrum of resistance carried by the three Prunus $R$ genes previously reported, by evaluating them toward an isolate of the RKN species yet untested (Table 1). Because each of the genes completely inhibits nematode multiplication and gall formation of the RKN species that they control, we used the gall index rating criterion to phenotype individuals as resistant (R) or susceptible (S). All three genes have been evaluated to M. ethiopica. The RMja almond gene has also been assessed to $M$. arenaria and $M$. enterolobii. In addition, the RMia peach gene has been evaluated toward the isolate of $M$. javanica to assess whether it might have a partial effect on this latter species using M. incognita as a reference controlled RKN species. To state whether a given gene controls a particular species, we genotyped a number of individuals segregating for the gene studied using appropriate markers, then phenotyped these individuals for resistance to this RKN species. From the genotype-phenotype matching, we finally established whether this latter gene controls the RKN species represented by its tested isolate.

\section{MATERIALS AND METHODS}

Plant material. The plant material belonged to plum ( $M a$ gene), almond ( $R M j a$ gene), and peach (RMia gene). In plum, an $M a$ segregating cross between the $M a$-resistant accession P.2980 (heterozygous; Ma/ma) (Lecouls et al. 2004; Rubio-Cabetas et al. 1998) and the susceptible accession P.1090 (rootstock cultivar Myrocal; $\mathrm{ma} / \mathrm{ma}$ ) (D. Esmenjaud, unpublished) has been specifically produced for our study (Fig. 1). The F1 progeny segregated into the two classes $\mathrm{Ma} / \mathrm{ma}(\mathrm{R})$ and $\mathrm{ma} / \mathrm{ma}(\mathrm{S})$. Forty-one random individuals were genotyped at the seedling stage. All individuals were subsequently phenotyped for resistance to $M$. ethiopica (Table 2). The $M a$ heterozygous donor P.2175 ( $\mathrm{Ma} / \mathrm{ma}$ ) (Claverie et al. 2004a,b, 2011) and three other $M a$-susceptible accessions (homozygous recessive, $\mathrm{ma} / \mathrm{ma}$ ) P.2032, P.16.5, and P.1254 were also used as controls (Lecouls 2000; Lecouls et al. 1999).

In peach, a four-way cross involving two F1 hybrids obtained from four peach accessions was used (Fig. 1). The first F1 individual was $($ Pamir $\times$ Rubira) 140 (= PR140) between the two peach accessions Pamir and Rubira, both susceptible to $M$. arenaria, $M$. incognita, and M. javanica, and homozygous recessive for RMia (rmia/rmia). The second F1 cross was Montclar $\times$ Nemared between the peach Montclar, susceptible to these RKN species and homozygous recessive for RMia (rmia/rmia), and Nemared, resistant to $M$. incognita and $M$. arenaria and homozygous

TABLE 1. Status of the resistance spectrum of the three Prunus resistance genes ( $M a$ from plum, $R M j a$ from almond, and $R M i a$ from peach) before the study ${ }^{\mathrm{r}}$

\begin{tabular}{|c|c|c|c|c|c|c|}
\hline Resistance source (gene) & Genotype & Meloidogyne arenaria & M. incognita & M. javanica & M. ethiopica & M. enterolobii \\
\hline P.2175 \& P.2980 (Ma) & $\mathrm{Ma} / \mathrm{ma}$ & $\mathrm{R}^{\mathrm{s}}$ & $\mathrm{R}^{\mathrm{t}}$ & $\mathrm{R}^{\mathrm{t}}$ & - & $\mathrm{R}^{\mathrm{u}}$ \\
\hline Alnem1 (RMja) & $R M j a / R M j a$ & - & $\mathrm{S}^{\mathrm{v}}$ & $\mathrm{R}^{\mathrm{w}}$ & - & - \\
\hline Nemared (RMia) & RMia/RMia & $\mathrm{R}^{\mathrm{x}}$ & $\mathrm{R}^{\mathrm{y}}$ & - & - & $\mathrm{S}^{\mathrm{z}}$ \\
\hline
\end{tabular}

${ }_{\mathrm{r}} \mathrm{R}=$ resistant, $\mathrm{S}=$ susceptible, and - = unknown.

s Esmenjaud et al. 1996.

t Lecouls et al. 1997.

u Rubio-Cabetas et al. 1999.

v Scotto La Massese et al. 1984

w Kochba and Spiegel-Roy 1975; Esmenjaud et al. 2009; Van Ghelder et al. 2010.

${ }^{x}$ Claverie et al. 2004a.

y Claverie et al. 2004a; Esmenjaud et al. 2009; Duval et al. 2014.

z Khallouk 2013.

\section{Myrobalan plum}

\begin{tabular}{lcc}
\hline P.2980 & x & P.1090 \\
& ma/ma & \\
& &
\end{tabular}

F1 Myro

Segregating into 2 classes:

$$
\text { - Ma/ma }
$$$$
\text { - } m a / m a
$$

Peach

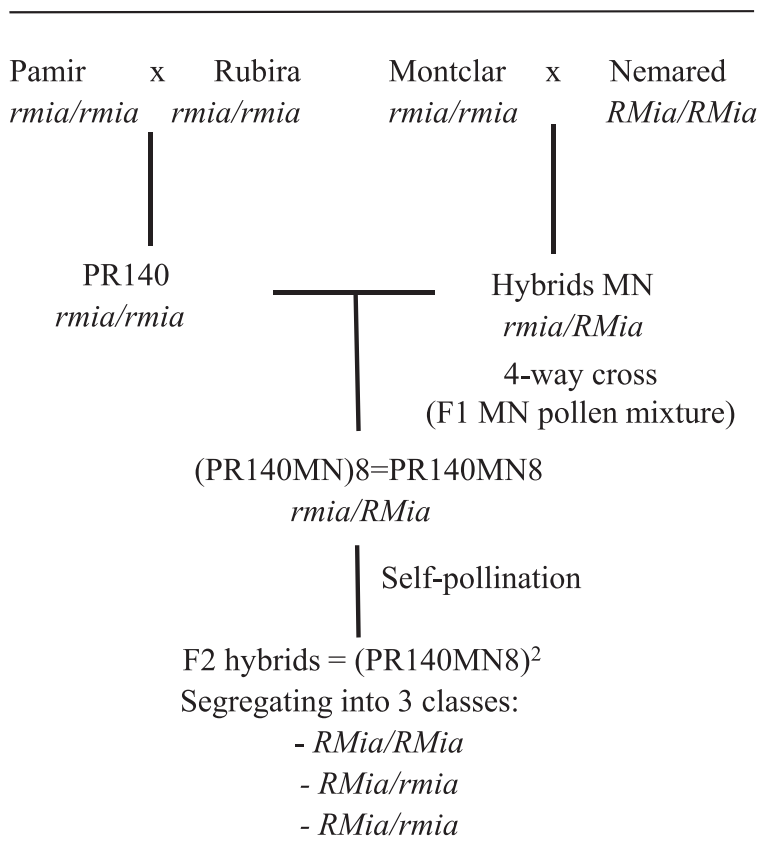

Almond

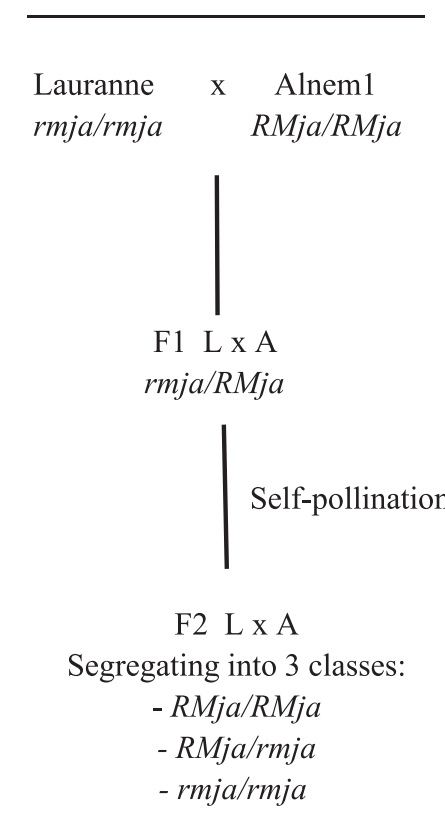

Fig. 1. Plant materials from Myrobalan plum, peach, and almond and the different crosses used in the study. 
dominant for RMia (RMia/RMia). The four-way offspring was created for this study by crossing the female hybrid PR140 and a mixture of the pollen from $51 \mathrm{~F} 1$ hybrids of Montclar $\times$ Nemared. The seedlings assessed were obtained from an F2 progeny of the hybrid number 8 of the PR $140 \times$ MN four-way cross (designated PR140MN8) and genotyped RMia/rmia using markers developed by Duval et al. (2014). These seedlings belonged to one of the three classes of individuals: RMia/RMia, RMia/rmia, and rmia/rmia (Fig. 1). In this objective, 104 (M. ethiopica), 79 (M. javanica), and 59 (M. incognita) random individuals were genotyped and subsequently phenotyped for resistance to RKNs (Tables 2 and 3).

In almond, an F2 progeny was obtained from the self-pollination of the F1 hybrid (Lauranne $\times$ Alnem1)135 (designated F2 LxA). The accession Lauranne is susceptible to RKN species and homozygous recessive for RMja (rmja/rmja) and the accession
Alnem1 is resistant to $M$. javanica and homozygous dominant for RMja (RMja/RMja) (Fig. 1). The individuals evaluated are a part of the offspring obtained for another study on the high-resolution mapping of the RMja gene (Van Ghelder et al. 2018). The F2 progeny segregated into three different genotypes for resistance (RMja/RMja, RMja/rmja, and rmja/rmja). In all, 89 (M. ethiopica), 69 (M. enterolobii), and 100 (M. arenaria) random individuals were genotyped and subsequently phenotyped for resistance to RKNs (Tables 2 and 4).

Nematode isolates. Five isolates were used: one from each of the three predominant species $M$. arenaria, $M$. incognita, and $M$. javanica; one from the species M. ethiopica; and one from the species M. enterolobii. For the three predominant RKNs, these were the isolates $M$. arenaria 'Six-Fours' from Six-Fours (Provence, France), M. incognita 'Calissane' from Calissanne (Provence,

TABLE 2. Evaluation of resistance to the root-knot nematode (RKN) species Meloidogyne ethiopica (isolate Nancagua, Chile) in reference, parental, and segregating materials for the Ma, RMia, and RMja genes ${ }^{\mathrm{t}}$

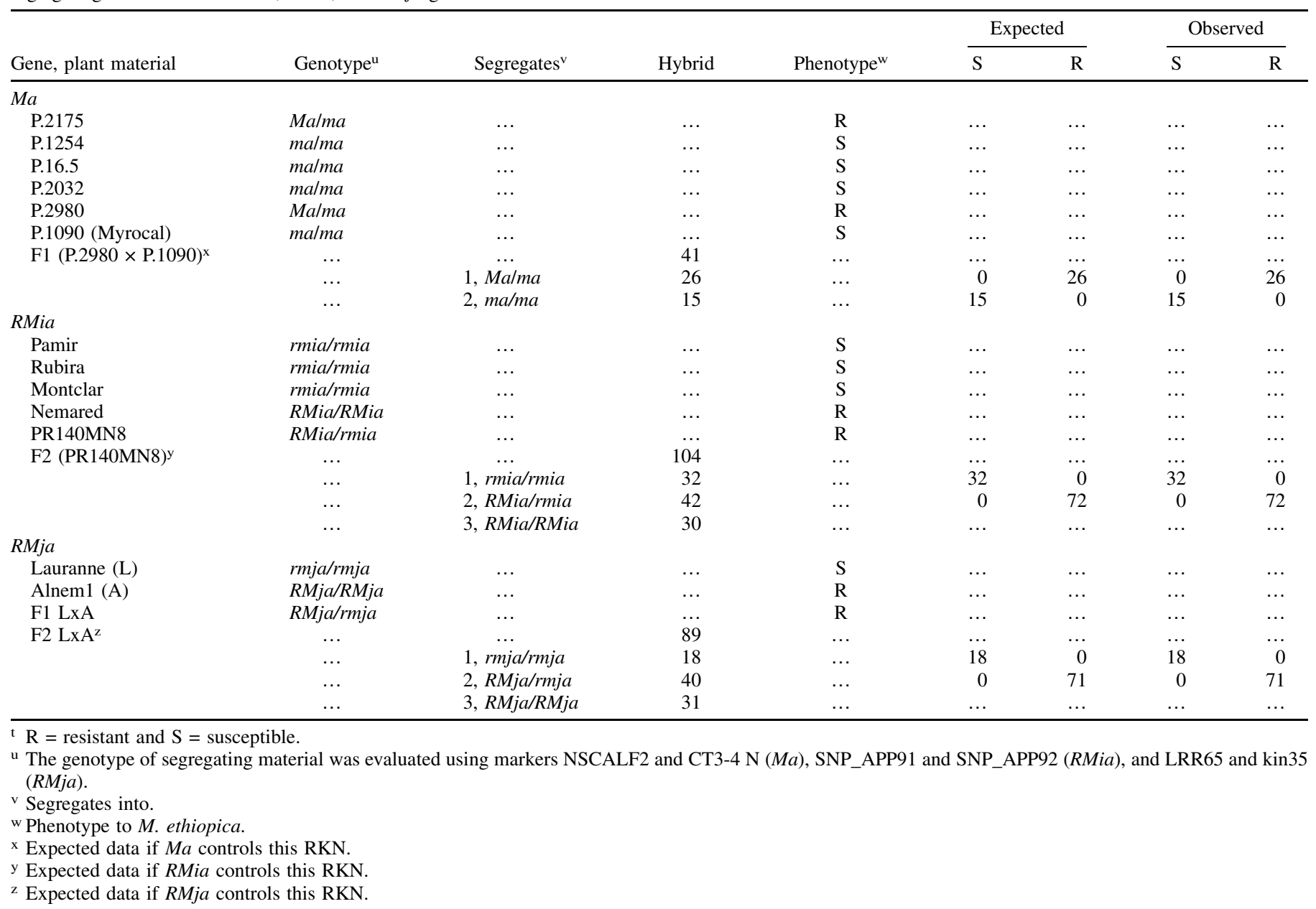

TABLE 3. Numbers of individuals and their resistance phenotype, gall index, collar diameter, BS root and top weights, after evaluation to the root-knot nematode species Meloidogyne javanica (M. jav, isolate Higuera, Cataluna) using M. incognita (M. inc, isolate Calissanne, France) as a control in the four-way cross PR140MN8 segregating for the RMia gene ${ }^{y}$

\begin{tabular}{|c|c|c|c|c|c|c|c|c|c|c|c|}
\hline \multirow[b]{2}{*}{ Class } & \multirow[b]{2}{*}{ Genotype $^{z}$} & \multicolumn{2}{|c|}{$\begin{array}{l}\text { Number of } \\
\text { individuals }\end{array}$} & \multicolumn{2}{|c|}{ Phenotype } & \multicolumn{2}{|c|}{ Gall index (0-5) } & \multicolumn{2}{|c|}{$\begin{array}{l}\text { Collar diameter } \\
(\mathrm{mm})\end{array}$} & \multirow{2}{*}{$\frac{\text { Root weight }(\mathrm{g})}{M . j a v}$} & \multirow{2}{*}{$\frac{\text { Top weight }(\mathrm{g})}{M \cdot j a v}$} \\
\hline & & M. jav & $\overline{M . \text { inc }}$ & M. jav & M. inc & M. jav & $\overline{\text { M. inc }}$ & M. jav & $\overline{\text { M. inc }}$ & & \\
\hline 1, Homozygous S & rmia/rmia & 30 & 17 & All S & All S & $1.83 \mathrm{a}$ & $3.12 \mathrm{a}$ & $8.15 \mathrm{a}$ & $7.53 \mathrm{~b}$ & $54.7 \mathrm{a}$ & $24.7 \mathrm{a}$ \\
\hline 3, Homozygous R & RMia/RMia & 19 & 17 & All S & All R & $1.89 \mathrm{a}$ & $0.18 \mathrm{~b}$ & $8.23 \mathrm{a}$ & $8.88 \mathrm{a}$ & $56.2 \mathrm{a}$ & $25.5 \mathrm{a}$ \\
\hline
\end{tabular}

${ }^{\mathrm{y}} \mathrm{R}=$ resistant and $\mathrm{S}=$ susceptible. In the same column, numbers followed by the same letter are not significantly different according to the least significant difference Fisher test at $P \leq 0.05$ (analysis of variance statistical analysis).

z The genotype of segregating material was evaluated using RMia markers SNP_APP91 and SNP_APP92. 
France), and M. javanica 'Higuera' from Cabrils (Cataluna, Spain). For M. ethiopica, we used the isolate Nancagua from this locality in the VI Region of Chile. This isolate has been evaluated on the peach Nemaguard and Pomona and identified as belonging to the 3 most aggressive and not statistically different isolates out of 15 isolates evaluated (Meza et al. 2016). We also used the isolate M. enterolobii 'Godet' from Godet in the Guadeloupe Island (French West Indies, France). All five isolates had been reared from a single egg mass. The isolates were maintained on tomato (Lycopersicon esculentum) cultivar St. Pierre. Their specific identity was verified before the experiments via their isoesterase profile (Esbenshade and Triantaphyllou 1985, 1990; Janati et al. 1982).

Greenhouse phenotyping method using a high- and durable-inoculum pressure test. Experiments were implemented over 3 years in three independent annual evaluations performed from March to November. Each year, we used a greenhouse phenotyping method based on a high- and durable-inoculum pressure test, as described hereafter. In March, plantlets obtained from 2- to 3-month-old seed (peach and almond) or semihardwood cuttings from the previous autumn (parental and reference materials from plum) were repotted in pairs into 5-liter containers filled with a nematode-free sandy substrate. Evaluations for RKN resistance were performed with a method derived from Esmenjaud et al. (1992). All of the pots were placed on iron benches in the greenhouse, irrigated individually every 2 days, and grown until harvested for rating at a mean temperature of $25^{\circ} \mathrm{C}$ (extremes of 20 to $30^{\circ} \mathrm{C}$ ). RKN inoculation was performed as follows. At mid-March, tomato plantlets grown in $250-\mathrm{ml}$ plastic pots were inoculated with 50024 - to 72-h-old juveniles (infective stage; J2), obtained from the INRA collection. Each of the RKN isolates was suspended in water and deposited into two holes, $2 \mathrm{~cm}$ deep and $2 \mathrm{~cm}$ from the stem. At mid-May, the top parts of tomato plants were cut and removed and one soil and root system content was transplanted into each Prunus pot. Pots inoculated with the same Meloidogyne sp. were grouped on the same greenhouse bench to avoid cross contaminations. Plants of the different genotypes (RR, $\mathrm{RS}$, or SS) for each gene were arranged in a randomized design.

Rating of RKN resistance for $R$ or $S$ phenotyping. In autumn, 5 months after inoculation with galled tomato, Prunus plants were harvested, carefully washed free of soil, and given a root gall index rating according to the 0 -to-5 scale (Barker 1985), where
$0=$ no gall and $1=1$ to $10 \%, 2=11$ to $30 \%, 3=31$ to $70 \%, 4=71$ to $90 \%$, and $5>90 \%$ of root system galled. This rating was completed with 0.5 steps when galling was intermediate between two categories. This rating procedure has been established in a previous study, which demonstrated that gall index was highly significantly correlated with the $\log _{10}(x+1)$ transformed numbers of the different nematode stages in the roots (Esmenjaud et al. 1992). When plants exhibited 1 to 5 incipient galls spread throughout the roots, these few galls were dissected to identify the fixed nematode stages (third and fourth developmental stages, female adults) and, thus, state whether the nematodes were the causal agent of the gall symptoms or not. Only in the positive case were they rated 0.1 (= $1 \mathrm{RKN}$ gall visually confirmed) or 0.5 (=2 to $5 \mathrm{RKN}$ galls visually confirmed) to refine the classification. Individuals from a segregating progeny (together with the parental and reference accessions) were classified into either the $\mathrm{R}(0 \leq$ gall index $\leq 0.5)$ or the $\mathrm{S}$ (gall index $>0.5$ ) phenotypes.

In the experiment that aimed to evaluate a quantitative effect of the isolate of M. javanica on plants carrying the RMia peach gene, harvested plants were rated for their collar diameter and their total root and aerial part weights (parts below and above the collar, respectively). In this experiment, additional plants were used as controls for resistance to $M$. incognita (RMia gene) and rated for their gall index and collar diameter (Table 3).

Molecular genotyping of plant material using resistance markers for each gene. Young terminal leaves of each seedling were collected as needed in the early spring and kept at $-80^{\circ} \mathrm{C}$ until DNA extraction. Total genomic DNA was isolated using the Dneasy kit (Qiagen). Polymerase chain reaction (PCR) assays were performed in a $12-\mu \mathrm{l}$ final volume containing $10 \mathrm{ng}$ of genomic DNA, $0.25 \mathrm{U}$ of Taq polymerase (Promega Corp.), $0.2 \mu \mathrm{M}$ each primer, $0.2 \mathrm{mM}$ each dNTP (Promega Corp.), $1.5 \mathrm{mM} \mathrm{MgCl}_{2}$, and $1 \times$ reaction buffer provided with the enzyme. PCR conditions were as follows: $94^{\circ} \mathrm{C}$ for $4 \mathrm{~min}$; then, 35 cycles of $94^{\circ} \mathrm{C}$ for $45 \mathrm{~s}$, annealing temperature of the primers for $45 \mathrm{~s}$, and $72^{\circ} \mathrm{C}$ for $45 \mathrm{~s}$; and, finally, $72^{\circ} \mathrm{C}$ for $4 \mathrm{~min}$. Amplifications were performed in a Mastercycler ep gradient thermal cycler (Eppendorf $\mathrm{GmbH}$ ). Markers were first screened using the parents and then using a set of individuals from the progeny to establish segregation patterns. The PCR products were separated on a $2.5 \%$ agarose gel or with a capillary sequencer (Applied Biosystems 3730 DNA Analyzer)

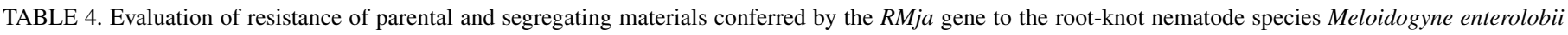
(isolate Godet, Guadeloupe) and M. arenaria (isolate Six-Fours, France) ${ }^{\mathrm{u}}$

\begin{tabular}{|c|c|c|c|c|c|c|c|c|}
\hline \multirow[b]{2}{*}{ Species, plant material } & \multirow[b]{2}{*}{ Genotype $^{\mathrm{v}}$} & \multirow[b]{2}{*}{ Segregatesw } & \multirow[b]{2}{*}{ Hybrid } & \multirow[b]{2}{*}{ Phenotype to ${ }^{x}$} & \multicolumn{2}{|c|}{ Expected } & \multicolumn{2}{|c|}{ Observed } \\
\hline & & & & & $\mathrm{S}$ & $\mathrm{R}$ & $\mathrm{S}$ & $\mathrm{R}$ \\
\hline \multicolumn{9}{|l|}{ M. enterolobii } \\
\hline Lauranne (L) & rmja/rmja & $\ldots$ & $\ldots$ & $\mathrm{S}$ & $\ldots$ & $\ldots$ & $\ldots$ & $\ldots$ \\
\hline Alnem1 (A) & $R M j a / R M j a$ & $\ldots$ & $\ldots$ & $\mathrm{R}$ & $\ldots$ & $\ldots$ & $\ldots$ & $\ldots$ \\
\hline F1 LxA & RMja/rmja & $\ldots$ & $\ldots$ & $\mathrm{R}$ & $\ldots$ & $\ldots$ & $\ldots$ & $\ldots$ \\
\hline \multirow[t]{4}{*}{$\mathrm{F} 2 \mathrm{LxA}^{\mathrm{y}}$} & $\ldots$ & $\ldots$ & 69 & $\ldots$ & $\ldots$ & $\ldots$ & $\ldots$ & $\ldots$ \\
\hline & $\ldots$ & 1, rmja/rmja & 20 & $\ldots$ & 20 & 0 & 20 & 0 \\
\hline & $\ldots$ & 2, RMja/rmja & 42 & $\ldots$ & 0 & 49 & 0 & 49 \\
\hline & $\ldots$ & 3, RMja/RMja & 7 & $\ldots$ & $\ldots$ & $\ldots$ & $\ldots$ & $\ldots$ \\
\hline \multicolumn{9}{|l|}{ M. arenaria } \\
\hline Lauranne (L) & rmja/rmja & $\ldots$ & $\ldots$ & $\mathrm{S}$ & $\ldots$ & $\ldots$ & $\ldots$ & $\ldots$ \\
\hline Alnem1 (A) & $R M j a / R M j a$ & $\ldots$ & $\ldots$ & $\mathrm{R}$ & $\ldots$ & $\ldots$ & $\ldots$ & $\ldots$ \\
\hline F1 LxA & RMja/rmja & $\ldots$ & $\ldots$ & $\mathrm{R}$ & $\ldots$ & $\ldots$ & $\ldots$ & $\ldots$ \\
\hline \multirow[t]{4}{*}{$\mathrm{F} 2 \mathrm{LxA}^{\mathrm{z}}$} & $\ldots$ & $\ldots$ & 100 & $\ldots$ & $\ldots$ & $\ldots$ & $\ldots$ & $\ldots$ \\
\hline & $\ldots$ & 1, rmja/rmja & 30 & $\ldots$ & 30 & 0 & 30 & 0 \\
\hline & $\ldots$ & 2, RMja/rmja & 47 & $\ldots$ & 0 & 70 & 0 & 70 \\
\hline & $\ldots$ & 3, RMja/RMja & 23 & $\ldots$ & $\ldots$ & $\ldots$ & $\ldots$ & $\ldots$ \\
\hline
\end{tabular}

u $\mathrm{R}=$ resistant and $\mathrm{S}=$ susceptible.

v The genotype of segregating material was evaluated using RMja markers LRR65 and kin35.

w Segregates into.

x Phenotype to M. enterolobii or M. arenaria.

y Expected data if the gene controls $M$. enterolobii.

${ }^{\mathrm{z}}$ Expected data if the gene controls $M$. arenaria. 
using nucleotides labeled with fluorochrome dyes. For the KASP assay, the reaction mixture was prepared according to the protocol described by LGC Genomics (https://www.lgcgroup.com/products/ kasp-genotyping-chemistry/\#.XDt67c1MHIU). Fluorescent endpoint genotyping was conducted using the 2103 EnVision Multilabel Reader (Perkin Elmer Company).

In the segregating plum material P.2980 $\times$ P.1090, individuals were genotyped and classified into two classes (heterozygous resistant, Ma/ma; and homozygous susceptible, $\mathrm{ma} / \mathrm{ma}$ ) using the sequence-characterized amplified region markers NSCALF2 and CT3-4 N located within the Ma gene genomic sequence (Claverie et al. 2011) (Supplementary Table S1). Similarly, in the segregating peach F2 PR140MN8 involving the RMia gene (Tables 2 and 3), individuals were genotyped and classified into three classes (homozygous resistant, RMia/RMia; heterozygous resistant, RMia/ rmia; and homozygous susceptible, rmia/rmia) using the flanking markers SNP_APP91 and SNP_APP92 (Duval et al. 2014). Finally, in the segregating almond F2 LxA progeny, individuals were genotyped and classified into the three classes $R M j a / R M j a, R M j a /$ rmja, and rmja/rmja using the flanking markers LRR65 and kin35 (Van Ghelder et al. 2018).

Statistical analysis. A part of the study aimed to evaluate whether the RMia gene, that controls the species M. incognita in particular, has a quantitative effect on $M$. javanica. In this objective, an analysis of variance using XLSTAT software (version 2014.5.03) was conducted for the quantitative criteria, gall index, collar diameter, root weight, and top weight. Significant differences between individuals were analyzed by the least significant difference Fisher test at $P \leq 0.05$. In this statistical analysis, all the individuals of the same genotypic group (RMia/RMia, RMia/rmia, and rmia/rmia) were considered as replicates (Table 3 ).

\section{RESULTS}

Study of the resistance to $M$. ethiopica. Each of the three genes Ma, RMia, and RMja has been evaluated toward the isolate of this RKN species. Molecular markers of the Ma, RMia, and RMja genes were used to genotype the segregating seedlings of their respective progenies.

$M a$ markers ranged the seedlings from the F1 cross P.2980 $\times$ P.1090 into one of the two genotypic classes, $\mathrm{Ma} / \mathrm{ma}$ or $\mathrm{ma} / \mathrm{ma}$. The phenotyping evaluation based on gall index ratings allowed distributing both the parental and F1 hybrid individuals into the R or $\mathrm{S}$ classes. Gall indices of resistant individuals ranged from 0 to 0.5 whereas gall indices of susceptible individuals were $\geq 1$. From the 41 total individuals of the progeny evaluated to $M$. ethiopica, all $26 \mathrm{Ma} / \mathrm{ma}$ individuals were resistant whereas all $15 \mathrm{ma} / \mathrm{ma}$ individuals were susceptible (Table 2). Consequently, individuals classified with $\mathrm{Ma}$ markers into the R heterozygous class $\mathrm{Ma} / \mathrm{ma}$ were resistant and individuals classified with $M a$ markers into the $\mathrm{S}$ genotypic class $\mathrm{ma} / \mathrm{ma}$ were susceptible.

$R M i a-$ and $R M j a$-specific markers ranged the segregating seedlings from the F2 crosses, PR140MN8 (104 individuals) and LxA (89 individuals), into one of the three genotypic classes RMia/ RMia, RMia/rmia, and rmia/rmia or RMja/RMja, RMja/rmja, and rmja/rmja, respectively. In parental and F2 hybrid materials from each cross, the high and durable inoculum pressure provided by the test allowed a clear classification of individuals into either the $\mathrm{R}(0 \leq$ gall index $\leq 0.5$ ) or the $S$ (gall index $\geq 1.0$ ) phenotypic groups. From the 104 total individuals of the F2 PR140MN8, all 30 RMia/RMia individuals and all $42 \mathrm{RMia} / \mathrm{rmia}$ individuals were resistant whereas all $32 \mathrm{rmia} / \mathrm{rmia}$ individuals were susceptible (Table 2). Consequently, individuals classified with RMia markers into the two R genotypic classes RMia/Rmia and RMia/rmia were resistant and individuals classified with $R M i a$ markers into the $\mathrm{S}$ genotypic class rmia/rmia were susceptible. Similarly, from the 89 total individuals of the F2 LxA progeny evaluated for resistance to M. ethiopica, all $31 \mathrm{RMja} / \mathrm{RMja}$ individuals and all $40 \mathrm{RMja} / \mathrm{rmja}$ individuals were resistant and all $18 \mathrm{rmja} / \mathrm{rmja}$ individuals were susceptible (Table 2). In other words, individuals from the two R RMja genotypic classes were resistant and all individuals from the $\mathrm{S} R M j a$ genotypic class were susceptible.

Consequently the complete matching of the genotypic and phenotypic classes obtained for Ma, RMia, and RMja strongly suggests that each of the three genes controls the $M$. ethiopica isolate used.

Study of the activity of the RMja gene to $M$. enterolobii and $M$. arenaria. $R M j a$-specific markers were used to genotype the 169 total segregating seedlings of the F2 cross LxA intended for a phenotypic evaluation to either $M$. enterolobii (69 individuals) or $M$. arenaria (100 individuals) and ranged them into one of the three genotypic classes $R M j a / R M j a, R M j a / r m j a$, and rmja/rmja. Two phenotypic classes were identified in both parental and F2 materials because individuals were clearly split into either the R or $\mathrm{S}$ classes. From the 69 total individuals of the F2 progeny evaluated to M. enterolobii, all 7 RMja/RMja individuals and all 42 RMja/rmja individuals were resistant whereas all $20 \mathrm{rmja} / \mathrm{rmja}$ individuals were susceptible (Table 4). Thus, individuals from the two R RMja genotypic classes were resistant and individuals from the S RMja genotypic class were susceptible. From the 100 total individuals of the F2 progeny evaluated to $M$. arenaria, all $23 R M j a / R M j a$ individuals and all $47 \mathrm{RMja} / \mathrm{rmja}$ individuals were resistant whereas all $30 \mathrm{rmja} / \mathrm{rmja}$ individuals were susceptible (Table 4). Therefore, individuals from the two R RMja genotypic classes were resistant and individuals from the S RMja genotypic class were susceptible. The absence of any recombinant individuals to $M$. enterolobii and to $M$. arenaria strongly suggests that the $R M j a$ gene controls the isolates of both RKN species.

Evaluation of a putative quantitative effect of the RMia gene on M. javanica. Because the RMia gene confers resistance to $M$. incognita, this RKN species was used as a positive control. We used a total number of 138 individuals from the F2 PR140MN8 progeny, which represents the largest progeny analyzed in this study. Of these, 79 individuals were evaluated for resistance to $M$. javanica and ranged into $30 \mathrm{rmia} / \mathrm{rmia}$ individuals, $30 \mathrm{RMia} /$ rmia individuals, and 19 RMia/RMia individuals (Table 3). The 59 other individuals were considered as controls for resistance to $M$. incognita and ranged into $17 \mathrm{rmia} / \mathrm{rmia}$ individuals, $25 \mathrm{RMia} /$ rmia individuals, and 17 RMia/RMia individuals (Table 3).

Gall index rating. As expected, the individuals of the three genotypic classes clearly segregated into two phenotypic groups after inoculation with the control species $M$. incognita: the R group (RMia/RMia and RMia/rmia genotypes) and the S group (rmia/rmia genotype). For this RKN species, mean gall index ratings of the two genotypic R classes (RMia/RMia and RMia/rmia) were not different but both differed significantly from the $\mathrm{S}$ class (rmia/rmia) (Table 3). By contrast, all 79 individuals evaluated to M. javanica were susceptible, whatever the genotypic class they belonged to. Moreover, the mean gall index ratings of the three genotypic classes from the individuals evaluated to this RKN species were not statistically different, which indicated that the RMia genetic status has no effect on the RKN galling observed toward this species. Consequently, the RMia gene did not confer resistance to the $M$. javanica isolate used.

Collar diameter. This criterion has been retained to illustrate the putative effect of the nematodes on the development of the plants that carried or lacked the RMia gene. After inoculation with M. incognita, plants with RMia/RMia and RMia/rmia genotypes displayed a collar diameter statistically higher $(P \leq 0.05)$ than plants with a homozygous $S$ genotype, which confirms both the activity of the RMia gene on this RKN species and its completely dominant status. By contrast, no significant differences between the three genotypic classes were observed in the plants inoculated by $M$. javanica. This illustrates that plant development was not influenced by the presence of the RMia gene, which is in line with its absence of effect toward $M$. javanica. 
Root and top weights. These two criteria were measured only on the plants tested to $M$. javanica. For both criteria, our results showed no significant differences $(P \leq 0.05)$ between the three genotypic classes of the RMia gene. This result supplements previous data on gall index and collar diameter ratings and may be related to RMia gene's lack of activity against the $M$. javanica isolate used.

\section{DISCUSSION}

We showed that the matching of the phenotypic and genotypic classes of the three genes for resistance to the RKN species evaluated was either complete (Ma, RMja, and RMia with M. ethiopica and RMja with M. arenaria and M. enterolobii) or null (RMia with $M$. javanica). No intermediate situation (i.e., a partial matching between these classes) occurred. Thus, our evaluation stated unambiguously whether a given gene is active against a given RKN isolate. In some tests, susceptible seedlings may occasionally escape the attacks, which generates false-resistant individuals. The high and durable inoculum pressure provided in our experimental procedure permitted us to avoid this major drawback by reducing considerably the risk of occurrence of misevaluated plants.

Nevertheless, our phenotypic results only allow a relative location of the $\mathrm{R}$ factor due to the limited number of segregating individuals (ranging from 41 to 104 for all crosses considered). Thus, theoretically, we cannot exclude the hypothesis that another closely linked factor actually controls the RKN species studied. In the study that confronts the $M a$ gene to the RKN isolates of M. ethiopica, the total number of individuals is 41 . This implies that the precision of the location of the genetic factor is approximately 2.5 centimorgans $(\mathrm{cM})$ and another $R$ factor located in an interval smaller than $2.5 \mathrm{cM}$ on each side of the gene could control it. Nevertheless, in this case, the $M a$ gene already controls five other RKN species, which is presumably not in line with the existence of an additional factor that would specifically control this species. For RMja-M. ethiopica and M. enterolobii, it had been recently shown that this gene is presumably an ortholog of $M a$ (Van Ghelder et al. 2018 ), which strongly suggests that no other $R$ factor is involved. Finally in RMia-M. ethiopica, numbers are the highest of our experiments (104 segregants) (i.e., allowing a precision of $\pm 1 \mathrm{cM}$ ), which limits the possible occurrence of another $R$ factor.

Consequently, our further discussion on the spectrum of the three Prunus $R$ genes Ma, RMia, and RMja will be founded on the hypothesis that these genes are alone in their respective chromosomal locations; or, in other words, that no other major $R$ gene closely linked to them is active against the RKN species studied here. Our comments will also support the premise that the isolate tested in each species is representative of the behavior of its RKN species. On this basis, our results are particularly interesting because they bring new information about the activity of all three $R$ genes to the five RKN species considered. The $M a$ gene was already known as controlling the five species $M$. arenaria, $M$. incognita, $M$. javanica, M. floridensis, and M. enterolobii (Lecouls 2000; Lecouls et al. 1997; Rubio-Cabetas et al. 1999). We showed that it also controls M. ethiopica, which confirms its complete spectrum against tested RKNs. This large spectrum tends toward a full resistance to all RKN species, which would be unique in plants thus far. This feature suggests that the $M a$ gene recognizes (directly or indirectly) a component which would be common to most if not all RKN species rather than several specific components. Thus, Prunus rootstocks carrying $M a$ may be suitable to use worldwide for
RKN resistance in any stone-fruit-producing regions. Regarding the $R M j a$ gene, we have completed previous results with the evaluation of its spectrum to three additional RKN species: $M$. arenaria, $M$. enterolobii, and $M$. ethiopica. Previous evaluations had shown that the Alnem sources (Alnem1, Alnem88, and Alnem201) controlled M. arenaria (Scotto La Massese et al. 1984) but the genetics of this resistance was ignored. We have established here that this resistance is conferred by $R M j a$ (i.e., by the same gene initially described as controlling M. javanica) (Van Ghelder et al. 2010). Interestingly, the $R M j a$ spectrum of resistance is close to the spectrum of $M a$. This enhanced range of resistance conferred by the $R M j a$ gene increases the global area where rootstocks carrying this almond gene would be able to face RKN attacks. Indeed, the major species $M$. arenaria has a very wide distribution and is present throughout the world in all regions benefitting from a Mediterranean climate. By contrast, the species M. ethiopica has a more restricted distribution area but is prevalent in Chile (Carneiro et al. 2007a; Meza et al. 2016) and also widely detected in Brazil (Carneiro et al. 2004; Correa et al. 2014). In addition, $R M j a$ is the second gene after $M a$ to confer resistance to the emerging aggressive species M. enterolobii. Unlike RMia or the $M i-1$ gene from tomato (Saucet et al. 2016), we showed that RMja fully controls the M. enterolobii isolate tested. Finally, our assessment focused on the RMia gene and evaluated it to $M$. ethiopica and M. javanica. We showed that this gene, like $M a$ and $R M$ ja, controls $M$. ethiopica. Having available several major $R$ genes to M. ethiopica is encouraging for the successful management of this RKN species.

Our study has also unraveled the interaction between the RMia peach gene and $M$. javanica. This gene has been shown to confer resistance to $M$. incognita and $M$. arenaria (Esmenjaud et al. 1997, 2009) but its status regarding $M$. javanica was unknown. Studies on the histological mechanisms of resistance in Nemaguard and its derived Nemared rootstock (Ramming and Tanner 1983) established that these plant materials control certain $M$. javanica populations despite the formation of small galls. A "walling-off" process isolating young giant cells from neighboring cells and preventing the maturation of the females in the infection site has been described (Malo 1967; Marull et al. 1994; Meyer 1978). The genetic determinants controlling $M$. javanica in Nemaguard and Nemared remain to be identified. Several genetic hypotheses such as two independent genes (Sharpe et al. 1969) or a single gene (Lu et al. 1998; Yoshida and Seike 1981) have been proposed. Other studies had suggested that $R$ genes for resistance to $M$. incognita might have a partial effect on M. javanica (Lu et al. 1998, 1999). Our data showed that the RMia gene does not confer resistance to the $M$. javanica isolate used here. This does not allow conclusions about the $M$. javanica species considered as a whole, and other isolates of this RKN species may be completely or partly controlled. Additionally, using M. incognita as an RKN species controlled by RMia confirmed the phenotypic effect of this gene, as illustrated with the parameter "collar diameter".

Our results point out the completely dominant status of the three Prunus genes, whatever the RKN species they face. This feature is particularly interesting for breeding because it allows growers to obtain completely resistant interspecific rootstock material from a single F1 hybridization between parents carrying different $R$ genes. We confirm that, in future Prunus breeding programs, resistance to all predominant species (i.e., $M$. arenaria, $M$. incognita, and $M$. javanica) will be possible by stacking the $R$ genes $R M i a$ (controlling $M$. arenaria and $M$. incognita) and RMja (controlling

TABLE 5. Spectrum for resistance conferred by the three Prunus resistance genes Ma, RMja, and RMia to six root-knot nematodes (Meloidogyne spp. ${ }^{\mathrm{z}}$

\begin{tabular}{|c|c|c|c|c|c|c|}
\hline Gene (resistance source) & Meloidogyne arenaria & M. incognita & M. javanica & M. ethiopica & M. floridensis & M. enterolobii \\
\hline$M a$ (plum, P.2175 and P.2980) & $\mathrm{R}$ & $\mathrm{R}$ & $\mathrm{R}$ & $\mathrm{R}$ & $\mathrm{R}$ & $\mathrm{R}$ \\
\hline RMja (almond, Alnem1) & $\mathrm{R}$ & $\mathrm{S}$ & $\mathrm{R}$ & $\mathrm{R}$ & $\mathrm{S}$ & $\mathrm{R}$ \\
\hline RMia (peach, Nemared) & $\mathrm{R}$ & $\mathrm{R}$ & $\mathrm{S}$ & $\mathrm{R}$ & $\mathrm{S}$ & $\mathrm{S}$ \\
\hline
\end{tabular}

${ }^{\mathrm{z}} \mathrm{R}=$ resistant and $\mathrm{S}=$ susceptible. 
M. javanica). Thus, using hybrid material Alnem $1 \times$ Nemared will guarantee the complete control of all three species. Given that the $M a$ plum gene also confers resistance to all three RKN species, pyramiding the three genes Ma, RMia, and RMja in the same rootstock will provide a double resistance layer to all three prevalent RKN species mentioned previously.

Finally, our results clearly establish the ability of each gene to control the different isolates tested. This allows us to draw a complete picture that summarizes the resistance conferred to six total Meloidogyne spp. (Table 5). Interestingly, this updated spectrum of resistance of Prunus genes shows that RMja differs from $M a$ by being inactive to $M$. incognita and $M$. floridensis. Phylogenetic data on these two parthenogenetic RKN species show that they are evolutionarily quite close (Holterman et al. 2009), even though M. floridensis is meiotic whereas M. incognita is mitotic. Furthermore $M a$ and $R M j a$, located on chromosome 7, are presumably orthologous TNL genes whereas RMia, located on chromosome 2 and for which candidate genes are also TNLs (Duval et al. 2014), is quite different. Given their highly probable orthologous status, their differential specificity may be attributable either to the gain of recognition of a ubiquitous effector from Meloidogyne spp. in $M a$ or to the loss of recognition of an effector specific to M. incognita and M. floridensis in RMja. Ultimately, understanding the resistance spectrum for each gene is a milestone for future studies aiming to decipher the molecular determinants involved in the resistance to RKNs.

\section{LITERATURE CITED}

Barker, K. R. 1985. Design of greenhouse and microplot experiments for evaluation of plant resistance to nematodes. Pages 103-113 in: Plant Nematology Laboratory Manual. B. M. Zuckerman, W. F. Mai, and M. B. Harrison, eds. University of Massachusetts Agriculture Experiment Station, Amherst.

Carneiro, R. G., Monaco, A. P., Moritz, M. P., Nakamura, K. C., and Scherer, A. 2006. Identification of Meloidogyne mayaguensis in guava and weeds, in loam soil in Parana State [Identificacao de Meloidogyne mayaguensis em goiabeira e em plantas invasoras, em solo argiloso, no Estado do Parana]. Nematol. Bras. 30:293-298.

Carneiro, R. M. D. G., Almeida, M. R. A., Cofcewicz, E. T., Magunacelaya, J. C., and Aballay, E. 2007a. Meloidogyne ethiopica, a major root-knot nematode parasitizing Vitis vinifera and other crops in Chile. Nematology 9: 633-639.

Carneiro, R. M. D. G., Cirotto, P. A., Quintanilha, A. P., Silva, D. B., and Carneiro, R. G. 2007b. Resistance to Meloidogyne mayaguensis in Psidium spp. accessions and their grafting compatibility with $P$. guajava cv. paluma. Fitopatol. Bras. 32:281-284.

Carneiro, R. M. D. G., Randig, O., Almeida, M. R. A., and Gomes, A. C. M. M. 2004. Additional information on Meloidogyne ethiopica Whitehead, 1968 (Tylenchida: Meloidogynidae), a root-knot nematode parasitising kiwi fruit and grapevine from Brazil and Chile. Nematology 6: 109-123.

Castagnone-Sereno, P. 2012. Meloidogyne enterolobii (= M. mayaguensis): Profile of an emerging, highly pathogenic, root-knot nematode species. Nematology 14:133-138.

Claverie, M., Bosselut, N., Lecouls, A. C., Voisin, R., Lafargue, B., Poizat, C., Kleinhentz, M., Laigret, F., Dirlewanger, E., and Esmenjaud, D. 2004a. Location of independent root-knot nematode resistance genes in plum and peach. Theor. Appl. Genet. 108:765-773.

Claverie, M., Dirlewanger, E., Bosselut, N., Van Ghelder, C., Voisin, R., Kleinhentz, M., Lafargue, B., Abad, P., Rosso, M. N., Chalhoub, B., and Esmenjaud, D. 2011. The $M a$ gene for complete-spectrum resistance to Meloidogyne species in Prunus is a TNL with a huge repeated C-terminal post-LRR region. Plant Physiol. 156:779-792.

Claverie, M., Dirlewanger, E., Cosson, P., Bosselut, N., Lecouls, A. C., Voisin, R., Kleinhentz, M., Lafargue, B., Caboche, M., Chalhoub, B., and Esmenjaud, D. 2004b. High-resolution mapping and chromosome landing at the root-knot nematode resistance locus $M a$ from Myrobalan plum using a large-insert BAC DNA library. Theor. Appl. Genet. 109:1318-1327.

Correa, V. R., Mattos, V. S., Almeida, M. R. A., Santos, M. F. A., Tigano, M. S., Castagnone-Sereno, P., and Carneiro, R. M. D. G. 2014. Genetic diversity of the root-knot nematode Meloidogyne ethiopica and development of a species-specific SCAR marker for its diagnosis. Plant Pathol. 63: 476-483.
De Guiran, G., and Netscher, G. 1970. Les nématodes du genre Meloidogyne parasites des cultures tropicales. Cahiers de l'ORSTOM. Ser. Biol. 3: $151-185$.

Duval, H., Hoerter, M., Polidori, J., Confolent, C., Masse, M., Moretti, A., Van Ghelder, C., and Esmenjaud, D. 2014. High-resolution mapping of the RMia gene for resistance to root-knot nematodes in peach. Tree Genet. Genomes 10:297-306.

Esbenshade, P. R., and Triantaphyllou, A. C. 1985. Electrophoretic methods for the study of root-knot nematode enzymes. Pages 115-123 in: An Advanced Treatise on Meloidogyne, Volume II: Methodology. K. R. Barker, C. C. Carter, and J. N. Sasser, eds. North Carolina State University Graphics, Raleigh.

Esbenshade, P. R., and Triantaphyllou, A. C. 1990. Isozyme phenotypes for the identification of Meloidogyne species. J. Nematol. 22:10-15.

Esmenjaud, D., Minot, J. C., Voisin, R., Bonnet, A., and Salesses, G. 1996. Inheritance of resistance to the root-knot nematode Meloidogyne arenaria in Myrobalan plum. Theor. Appl. Genet. 92:873-879.

Esmenjaud, D., Minot, J. C., Voisin, R., Pinochet, J., Simard, M. H., and Salesses, G. 1997. Differential response to root-knot nematodes in Prunus species and correlative genetic implications. J. Nematol. 29:370-380.

Esmenjaud, D., Scotto La Massese, C., Salesses, G., Minot, J. C., and Voisin, R. 1992. Method and criteria to evaluate resistance to Meloidogyne arenaria in Prunus cerasifera Ehr. Fundam. Appl. Nematol. 15:385-389.

Esmenjaud, D., Voisin, R., Van Ghelder, C., Bosselut, N., Lafargue, B., Di Vito, M., Dirlewanger, E., Poessel, J. L., and Kleinhentz, M. 2009. Genetic dissection of resistance to root-knot nematodes Meloidogyne spp. in plum, peach, almond, and apricot from various segregating interspecific Prunus progenies. Tree Genet. Genomes 5:279-289.

Handoo, Z. A., Nyczepir, A. P., Esmenjaud, D., van der Beek, J. G., Castagnone-Sereno, P., Carta, L. K., Skantar, A. M., and Higgins, J. A. 2004. Morphological, molecular, and differential-host characterization of Meloidogyne floridensis n. sp. (Nematoda: Meloidogynidae), a root-knot nematode parasitizing peach in Florida. J. Nematol. 36:20-35.

Holterman, M., Karssen, G., van den Elsen, S., van Megen, H., Bakker, J., and Helder, J. 2009. Small subunit rDNA-based phylogeny of the Tylenchida sheds light on relationships among some high-impact plant-parasitic nematodes and the evolution of plant feeding. Phytopathology 99:227-235.

Janati, A., Bergé, J. B., Triantaphyllou, A. C., and Dalmasso, A. 1982. Nouvelles données sur l'utilisation des isoestérases pour l'identification des Meloidogyne. Rev. Nematol. 5:147-154.

Jones, J. T., Haegeman, A., Danchin, E. G. J., Gaur, H. S., Helder, J., Jones, M. G. K., Kikuchi, T., Manzanilla-Lopez, R., Palomares-Rius, J. E., Wesemael, W. M. L., and Perry, R. N. 2013. Top 10 plant-parasitic nematodes in molecular plant pathology. Mol. Plant Pathol. 14:946-961.

Khallouk, S. 2013. Interaction Meloidogyne-Prunus: Contribution to the study of the histological mechanisms and of the durability of resistance. (In French). Ph.D. thesis, Montpellier SupAgro, Montpellier, France.

Kochba, J., and Spiegel-Roy, P. 1975. Inheritance of resistance to the root-knot nematode (Meloidogyne javanica Chitwood) in bitter almond progenies. Euphytica 24:453-457.

Lamberti, F. 1979. Economic importance of Meloidogyne spp. in subtropical and Mediterranean climates. Pages 341-357 in: Root-Knot Nematodes (Meloidogyne Species): Systematics, Biology and Control. F. Lamberti and C. E. Taylor, eds. Academic Press, New York.

Lecouls, A. C. 2000. Spectrum of activity and molecular markers of the Mal gene for resistance to RKN Meloidogyne spp. in Myrobalan plum. (In French). Ph.D. thesis, Aix-Marseille II, Marseille, France.

Lecouls, A. C., Bergougnoux, V., Rubio-Cabetas, M. J., Bosselut, N., Voisin, R., Poessel, J. L., Faurobert, M., Bonnet, A., Salesses, G., Dirlewanger, E., and Esmenjaud, D. 2004. Marker-assisted selection for the wide-spectrum resistance to root-knot nematodes conferred by the $M a$ gene from Myrobalan plum (Prunus cerasifera) in interspecific Prunus material. Mol. Breed. 13:113-124.

Lecouls, A. C., Rubio-Cabetas, M. J., Minot, J. C., Voisin, R., Bonnet, A., Salesses, G., Dirlewanger, E., and Esmenjaud, D. 1999. RAPD and SCAR markers linked to the Mal root-knot nematode resistance gene in Myrobalan plum (Prunus cerasifera Ehr.). Theor. Appl. Genet. 99:328-335.

Lecouls, A. C., Salesses, G., Minot, J. C., Voisin, R., Bonnet, A., and Esmenjaud, D. 1997. Spectrum of the Ma genes for resistance to Meloidogyne spp. in Myrobalan plum. Theor. Appl. Genet. 95:1325-1334.

Lu, Z. X., Sosinski, B., Reighard, G. L., Baird, W. V., and Abbott, A. G. 1998. Construction of a genetic linkage map and identification of AFLP markers for resistance to root-knot nematodes in peach rootstocks. Genome 41: 199-207.

Lu, Z. X., Sossey-Alaoui, K., Reighard, G. L., Baird, W. V., and Abbott, A. G. 1999. Development and characterization of a codominant marker linked to root-knot nematode resistance, and its application to peach rootstock breeding. Theor. Appl. Genet. 99:115-122.

Malo, S. E. 1967. Nature of resistance of 'Okinawa' and 'Nemaguard' peach to the root-knot nematode Meloidogyne javanica. Proc. Am. Soc. Hortic. Sci. 90:39-46. 
Marull, J., Pinochet, J., Felipe, A., and Cenis, J. L. 1994. Resistance verification in Prunus selections to a mixture of 13 Meloidogyne isolates and resistance mechanisms of a peach-almond hybrid to $M$. javanica. Fundam. Appl. Nematol. 17:85-92.

Meyer, A. J. 1978. Why is 'Nemaguard' rootstock resistant to root-knot nematodes (Meloidogyne spp.) and 'Kakamas' susceptible? Deciduous Fruit Grower 28:66-72.

Meza, P., Soto, B., Rojas, L., and Esmenjaud, D. 2016. Identification of Meloidogyne species from the Central Valley of Chile and interaction with stone fruit rootstocks. Plant Dis. 100:1358-1363.

Rammah, A., and Hirschmann, H. 1988. Meloidogyne mayaguensis n. sp. (Meloidogynidae), a root-knot nematode from Puerto-Rico. J. Nematol. 20:58-69.

Ramming, D. W., and Tanner, O. 1983. Nemared peach rootstock. HortScience 18:376.

Rehder, A. 1947. Manual of Cultivated Trees and Shrubs. Macmillan Company, New York.

Rubio-Cabetas, M. J., Lecouls, A. C., Salesses, G., Bonnet, A., Minot, J. C., Voisin, R., and Esmenjaud, D. 1998. Evidence of a new gene for high resistance to Meloidogyne spp. in Myrobalan plum, Prunus cerasifera. Plant Breed. 117:567-571.

Rubio-Cabetas, M. J., Minot, J. C., Voisin, R., Esmenjaud, D., Salesses, G., and Bonnet, A. 1999. Resistance response of the $M a$ genes from 'Myrobalan' plum to Meloidogyne hapla and M. mayaguensis. HortScience 34:1266-1268.

Salesses, G., Grasselly, C., Renaud, R., and Claverie, J. 1992. Les porte-greffe des espèces fruitières à noyau du genre Prunus. Pages 605-650 in: Amélioration des espèces végétales cultivées: Objectifs et critères de sélection. A. Gallais and H. Bannerot, eds. INRA Editions, Paris.

Saucet, S. B., Van Ghelder, C., Abad, P., Duval, H., and Esmenjaud, D. 2016. Resistance to root-knot nematodes Meloidogyne spp. in woody plants. New Phytol. 211:41-56.

Scotto La Massese, C., Grasselly, C., Minot, J. C., and Voisin, R. 1984. Différence de comportement de 23 clones et hybrides de Prunus à l'égard de quatre espèces de Meloidogyne. Rev. Nematol. 7:265-270.
Sharpe, R. H., Hesse, C. O., Lownsbery, B. A., Perry, V. G., and Hansen, C. J. 1969. Breeding peaches for root-knot nematode resistance. J. Am. Soc. Hortic. Sci. 94:209-212.

Triantaphyllou, A. C. 1985. Cytogenetics, cytotaxonomy and phylogeny of root-knot nematodes. Pages 113-126 in: An Advanced Treatise on Meloidogyne, Volume I: Biology and Control. J. N. Sasser, and C. C. Carter, eds. North Carolina State University Graphics, Raleigh.

Van Ghelder, C., Esmenjaud, D., Callot, C., Dubois, E., Mazier, M., and Duval, D. 2018. Ma orthologous genes in Prunus spp. shed light on a noteworthy NBS-LRR cluster conferring differential resistance to root-knot nematodes. Front. Plant Sci. 9:1269.

Van Ghelder, C., Lafargue, B., Dirlewanger, E., Ouassa, A., Voisin, R., Polidori, J., Kleinhentz, M., and Esmenjaud, D. 2010. Characterization of the RMja gene for resistance to root-knot nematodes in almond: Spectrum, location, and interest for Prunus breeding. Tree Genet. Genomes 6:503-511.

Verde, I., Abbott, A. G., Scalabrin, S., Jung, S., Shu, S., Marroni, F., Zhebentyayeva, T., Dettori, M. T., Grimwood, J., Cattonaro, F., Zuccolo, A., Rossini, L., Jenkins, J., Vendramin, E., Meisel, L. A., Decroocq, V., Sosinski, B., Prochnik, S., Mitros, T., Policriti, A., Cipriani, G., Dondini, L., Ficklin, S., Goodstein, D. M., Xuan, P., Del Fabbro, C., Aramini, V., Copetti, D., Gonzalez, S., Horner, D. S., Falchi, R., Lucas, S., Mica, E., Maldonado, J., Lazzari, B., Bielenberg, D., Pirona, R., Miculan, M., Barakat, A., Testolin, R., Stella, A., Tartarini, S., Tonutti, P., Arus, P., Orellana, A., Wells, C., Main, D., Vizzotto, G., Silva, H., Salamini, F., Schmutz, J., Morgante, M., and Rokhsar, D. S. 2013. The high-quality draft genome of peach (Prunus persica) identifies unique patterns of genetic diversity, domestication and genome evolution. Nat. Genet. 45:487-494.

Yang, B., and Eisenback, J. D. 1983. Meloidogyne enterolobii n. sp. (Meloidogynidae), a root-knot nematode parasitizing pacara earpot tree in China. J. Nematol. 15:381-391.

Yoshida, M., and Seike, K. 1981. Breeding peach rootstocks resistant to rootknot nematode. 2. Breeding resistant rootstocks by hybridization. Bull. Fruit Tree Res. Stn. Yatabe Ibaraki Jpn. 8:31-34. 